\title{
Design of a New Family of Two-Dimensional Codes for Fiber-Optic CDMA Networks
}

\author{
E. S. Shivaleela, Kumar N. Sivarajan, Member, IEEE, and A. Selvarajan
}

\begin{abstract}
We report the design of a new family of twodimensional codes for fiber-optic CDMA networks. These newly designed temporal/spatial single-pulse-per-row (T/S SPR) codes have out-of-phase autocorrelation zero and cross correlation equal to one. Optical orthogonal codes (OOC's) have the lowest out-of-phase autocorrelation and cross-correlation values (both equal to one) among the one-dimensional codes. We compare the performance of our codes to the OOC's for a given probability of error. Experimentally we verify the autocorrelation and crosscorrelation properties of our codes and Park et al. [6] codes.
\end{abstract}

\section{INTRODUCTION}

$\mathbf{I}$ $\mathrm{N}$ local area networks (LAN's) the traffic being bursty, asynchronous multiplexing schemes are more efficient than synchronous multiplexing schemes. Fiber-optic code division multiple access (FO-CDMA) is one such scheme which is well suited for high speed LAN's. In FO-CDMA the optical signal is spread over a frequency band much wider than the minimum bandwidth required to send the information. The spreading of the signal is achieved by encoding it with a suitable sequence. To exploit the potential of FO-CDMA, optical signal processing is essential. Using fiber-optic tapped delay lines (FO-TDL) it is possible to optically encode/decode the chosen sequences.

Conventional bipolar codes used in electronic CDMA are not suitable for FO-CDMA networks using incoherent signal processing. Although coherent signal processing in FO-CDMA is possible in principle, it is not practical because of the difficulty in maintaining the correct phase at the high frequency of the optical carrier. Hence unipolar $\{0,1\}$ codes which have low out-of-phase autocorrelation and cross-correlation values are used for asynchronous FO-CDMA networks. Unipolar codes maintain low out-of-phase autocorrelation and cross correlation by minimizing the number of coincidences of ones rather than by cancellation as in bipolar codes. Hence, the number of ones in a unipolar code is less than the number of zeros.

The primary task of the receiver or decoder in a FOCDMA network is to recover the signal in the presence of other interfering signals. Hence codes suitable for FO-CDMA systems should have the following properties [1]:

Manuscript received April 25, 1997. This work was supported by the University Grants Commission, Government of India, under its Special Assistance Program. This paper was presented inpart at the NCC'95 [10] and NCC'96 [11] conferences.

The authors are with the Electrical Communication Engineering Department, Indian Institute of Science, Bangalore 560012 India.

Publisher Item Identifier S 0733-8724(98)01720-4.
- a code should be distinguishable from a shifted version of itself;

- a code should be distinguishable from a possibly shifted version of all other codes in the set.

The first condition implies that out-of-phase autocorrelation should be low for faster synchronization and also for low error probability. The second condition implies that cross correlation should be low for keeping the probability of error low due to multiple access interference.

Ideal orthogonality between the sequences cannot be obtained as the signals are unipolar in nature, that is, two signals cannot be added up to get zero as it is power addition. Hence codes with low out-of-phase autocorrelation and cross correlation are required to be designed.

One-dimensional (1-D) codes are characterized by $N\left(L_{T}, W, \lambda_{a}, \lambda_{c}\right)$ where $N$ is the number of codes, $L_{T}$ is the temporal length of the code, $W$ is the weight of the code (number of ones in the code), $\lambda_{a}$ is the peak out-of-phase autocorrelation value and $\lambda_{c}$ is the peak cross-correlation value. $L_{T}=T_{b} / T_{c}$ where $T_{b}$ is the bit time and $T_{c}$ is the chip time.

The autocorrelation for a 1-D code $x(t)$ is defined as follows:

$$
Z_{x, x}(l)=\sum_{n=0}^{L_{T}-1} x_{n} x_{(n+l) \bmod L_{T}} .
$$

$Z_{x, x}(l)$ satisfies

$$
Z_{x, x}(l) \begin{cases}=W & \text { if } l=0 \\ \leq \lambda_{a} & \text { if } 1 \leq l \leq L_{T}-1\end{cases}
$$

The cross correlation of 1-D codes $x(t)$ and $y(t)$ is defined as follows:

$$
Z_{x, y}(l)=\sum_{n=0}^{L_{T}-1} x_{n} y_{(n+l) \bmod L_{T}}
$$

$Z_{x, y}(l)$ satisfies

$$
Z_{x, y}(l) \leq \lambda_{c} \quad \text { if } 0 \leq l \leq L_{T}-1
$$

where $x(t)$ and $y(t)$ are two periodic 1-D codes and $L_{T}$ is the temporal length or the number of chips.

Several 1-D codes suitable for asynchronous FO-CDMA network have been reported in [1]-[5]. Among them OOC's have the lowest $\lambda_{a}$ and $\lambda_{c}$ values-both are equal to 1 . But the disadvantage of OOC's is that as the number of users or the weight of the code is increased, the length of the sequence increases rapidly. As a result of this, for a given chip width 


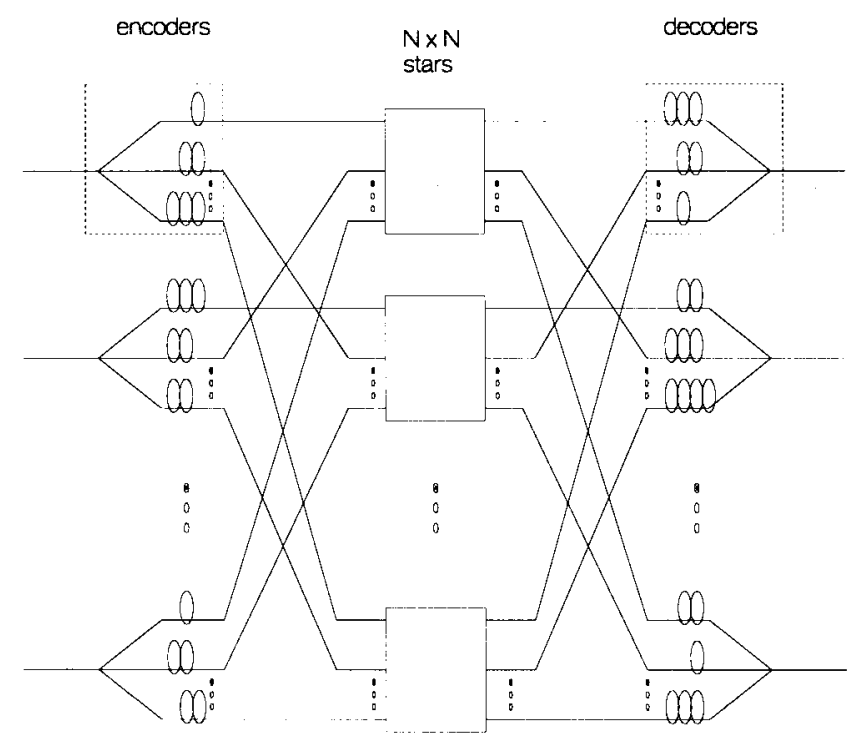

Fig. 1. Schematic diagram of a single-pulse-per-row temporal/spatial fiber-optic CDMA network.

the bit rate reduces which is not desirable. As a solution to this problem two-dimensional (2-D) coding is necessary. Park et al. [6] have demonstrated a T/S SPR incoherent, asynchronous FO-CDMA network. A schematic diagram of a T/S SPR network is as shown in Fig. 1. In Fig. 1, the number of spatial channels is equal to the number of distribution stars and the size of each star is equal to the number of users.

The advantage of T/S SPR networks is that single path losses are reduced as T/S SPR encoder (decoder) requires one $1 \times W$ coupler as opposed to temporal encoders which require two $1 \times W$ couplers. T/S SPR code correlation is energy efficient as there are no sidelobes in the autocorrelation. In incoherent signal processing, this property is unique to $\mathrm{T} / \mathrm{S}$ SPR codes because of the time-space correlation process.

Autocorrelation for a 2-D code $x(t)$ is defined as follows:

$$
Z_{x, x}(l)=\sum_{m=0}^{R-1}\left(\sum_{n=0}^{L_{T}-1} x_{m, n} x_{m,(n+l) \bmod L_{T}}\right) .
$$

$Z_{x, x}(l)$ satisfies

$$
Z_{x, x}(l) \begin{cases}=W & \text { if } l=0 \\ \leq \lambda_{a} & \text { if } 1 \leq l \leq L_{T}-1\end{cases}
$$

Cross correlation for 2-D codes $x(t)$ and $y(t)$ is defined as follows:

$$
Z_{x, y}(l)=\sum_{m=0}^{R-1}\left(\sum_{n=0}^{L_{T}-1} x_{m, n} y_{m,(n+l) \bmod L_{T}}\right) .
$$

$Z_{x, y}(l)$ satisfies,

$$
Z_{x, y}(l) \leq \lambda_{c} \quad \text { if } 0 \leq l \leq L_{T}-1
$$

where $x(t)$ and $y(t)$ are two periodic 2-D codes, $L_{T}$ is the temporal length and $R$ is the number of rows in the codes $x(t)$ or $y(t)$.

It can be shown that using the above definitions T/S SPR codes used in the demonstration by Park et al. [6] have $\lambda_{a}=0$ and $\lambda_{c}=2$. We have also verified experimentally that Park et al. codes have $\lambda_{c}=2$. Mendez et al. [7] have demonstrated multiple-pulses-per-row 2-D codes. Using the above definitons it can be shown that these multiple-pulses-row codes have $\lambda_{a}=2$ and $\lambda_{c}=2$.

In Section II of this paper, we describe the construction of a new family of 2-D codes which have the best known, nearly ideal, autocorrelation and cross-correlation properties for incoherent asynchronous FO-CDMA networks. The motivation for the design of new 2-D codes is to obtain the maximum number of codes with maximum weight and minimum length with the best possible autocorrelation and cross-correlation properties. For a given error performance the temporal lengths required for the newly designed codes and OOC's are compared in Section III. Also, the capacities of 1-D FO-CDMA networks employing OOC's and the 2-D T/S SPR networks employing the newly designed codes are compared. Experimental verification of the properties of the newly designed codes and Park et al. codes [6] are given in Section IV.

\section{DESIGN OF CODES}

In this section, we construct a new family of T/S SPR codes by an algebraic method. T/S SPR codes constructed by this method are slightly longer than the codes of Park et al. [6] and shorter than the codes of Mendez et al. [7]..$^{1}$ These new codes have improved cross-correlation properties over the other 2-D codes. The new codes are designed using the operation of addition modulo an integer. We name these codes as temporal/spatial addition modulo $L_{T}(\mathrm{~T} / \mathrm{S}$ AML) codes. T/S AML codes are a family of $N$ T/S SPR codes characterized by $N\left(L_{T}, R, p, W, \lambda_{a}, \lambda_{c}\right)$ where $L_{T}$ is the temporal length, $R$ is the number of rows which is equal to the number of spatial channels, $p$ is the number of pulses/row and is equal to one, $W$ is the weight of the code and is equal to $R \times p, \lambda_{a}$ is the outof-phase autocorrelation peak, and $\lambda_{c}$ is the cross-correlation peak.

A T/S AML code is represented by the nonzero column numbers in rows from $0,1, \cdots, R-1$. For example

$$
\begin{aligned}
& \text { Time } \rightarrow \\
& {\left[\begin{array}{l}
0 \\
1 \\
3 \\
2 \\
4
\end{array}\right] \text { represents }\left[\begin{array}{l}
10000 \\
01000 \\
00010 \\
00100 \\
00001
\end{array}\right] \begin{array}{ll}
S \\
p \\
c & \downarrow \\
e_{\text {. }} & \downarrow
\end{array}}
\end{aligned}
$$

The number of T/S AML codes that can be obtained for $L_{T}=W, \lambda_{a}=0$ and $\lambda_{c}=1$ depends on the type of the integer $L_{T}$. Alternatively, when $L_{T}=N, \lambda_{a}=0, \lambda_{c}=1$ and the maximum weight that can be obtained depends on the type of the integer $L_{T}$.

The position of ones in a T/S AML code $C_{i}$ is determined by one of the elements $j$ of the group of integers modulo $L_{T}$ (under addition) as follows:

$$
\begin{aligned}
& R_{0}(j)=0, \quad \text { for } j=0,1, \cdots, L_{T}-1 \\
& R_{i}(j)=\left(R_{(i-1)}(j)+j\right) \bmod \left(L_{T}\right), \quad 1 \leq i \leq L_{T}-1 \\
& { }^{1} \text { In [6] and [7] codes are given only for } N=4 .
\end{aligned}
$$


where

$$
C_{i}=\left[\begin{array}{c}
R_{o} \\
R_{1} \\
\vdots \\
R_{L_{T}-1}
\end{array}\right]
$$

and $R_{0}, R_{1}, \cdots, R_{L_{T}-1}$ represent the positions of ones in the rows $0,1, \cdots, L_{T}-1$, respectively. The element $j$ is called the generator of the code.

T/S AML codes are SPR codes and hence the out-of-phase autocorrelation is zero. In T/S AML codes when the distances between the ones in the respective rows of the two codes are distinct for all the rows, then $\lambda_{c}=1$ and this result is proved in the following theorem.

Theorem 1: $\lambda_{c}=1$ for any two T/S AML codes generated by $a$ and $c$ under addition modulo $L_{T}$, if $a-c=e$ is relatively prime to $L_{T}$.

Proof: Let $a$ and $c$ be the generators of two T/S AML codes under addition modulo $L_{T}$

$$
\begin{aligned}
& a x \equiv b\left(\bmod L_{T}\right) 0 \leq x \leq L_{T}-1 \\
& c x \equiv d\left(\bmod L_{T}\right) 0 \leq x \leq L_{T}-1 .
\end{aligned}
$$

The residues of (9) represent one code and that of (10) represent the other code. For the cross correlation to be one between any two T/S AML codes, the difference between the ones in the respective rows, for all the rows have to be distinct. $\mathrm{T} / \mathrm{S}$ AML codes have this property.

The difference between the elements of the codes generated by $a$ and $c$ is given by

$$
(a-c) x \equiv(b-d)\left(\bmod L_{T}\right) .
$$

The above equation can be written as

$$
e x \equiv f \bmod \left(L_{T}\right)
$$

where

$$
\begin{aligned}
& e=a-c, \quad \text { if } a \geq c \\
& e=(a-c)+L_{T}, \quad \text { if } a<c
\end{aligned}
$$

and

$$
\begin{aligned}
& f=b-d, \quad \text { if } b \geq d \\
& f=(b-d)+L_{T}, \quad \text { if } b<d .
\end{aligned}
$$

All the residues of (12) are distinct only if $e$ is relatively prime to $L_{T}$.

The number of T/S AML codes that can be constructed depends on the type of the integer $L_{T}$ and is analyzed in two cases:

- when $L_{T}$ is a composite number;

- when $L_{T}$ is a prime number.

Number of T/S AML codes that can be constructed is equal to the smallest prime factor of $L_{T}$, the length of the code. We prove this result in the following theorem.

Theorem 2: When $L_{T}$ is a composite number, $L_{T}=$ $W, \lambda_{a}=0$ and $\lambda_{c}=1$, the number of T/S AML codes that can be constructed is equal to the smallest prime factor of $L_{T}$.
Proof: Let $p$ be the smallest prime factor of $L_{T}$. Generators, $0 \leq j \leq L_{T}-1$, are grouped into $p$ groups such that the group $k$ contains those generators which have $k$ as the residue in the following equation:

$$
j \equiv k(\bmod p) 0 \leq k \leq p-1 .
$$

Each group contains $L_{T} / p$ elements. Because of Theorem 1 one generator from each group can be chosen to generate codes which satisfy $\lambda_{c}=1$. (Difference between two generators belonging to the same group $\equiv p$ which divides $L_{T}$.) Therefore, at most $p$ generators can be chosen, one from each group which proves the theorem.

Corollary: For $L_{T}$ prime, $L_{T}=W, \lambda_{a}=0$ and $\lambda_{c}=1$ the number of T/S AML codes that can be constructed is equal to $L_{T}$.

Proof: Follows from Theorem 2 since the smallest prime factor of $L_{T}$ is $L_{T}$.

We conjecture the following. The number of T/S SPR codes that can be constructed is equal to the smallest prime of factor of $L_{T}$, the length of the code when $\lambda_{c}=1$ and $L_{T}=W$. We prove this result when the length of the code $L_{T}$ is even.

Theorem 3: The number of T/S SPR codes that can be constructed is equal to two for even $L_{T}$, when $L_{T}=W$ and $\lambda_{c}=1$.

Proof: Let $L_{T}$ be an even number. Without loss of generality we assume the following two codes. The first code is represented by $L_{T}$ zeros and the second code by $x_{0}, x_{1}, \cdots, x_{L_{T}-1}$ distinct elements where $0 \leq x_{i} \leq L_{T}-1$. A valid third code should be represented by $L_{T}$ distinct elements because the difference between the elements of the third and the first codes have to be distinct. Let the third code be represented by $y_{0}, y_{1}, \cdots, y_{L_{T}-1}$ with $L_{T}$ distinct elements and $0 \leq y_{i} \leq L_{T}-1$.

Let $z_{i}$ be defined as follows:

$$
\begin{aligned}
& z_{i}=0, \quad \text { if } y_{i}=x_{i} \\
& z_{i}=y_{i}-x_{i}, \quad \text { if } y_{i}>x_{i} \\
& z_{i}=\left(y_{i}-x_{i}\right)+L_{T}, \quad \text { if } y_{i}<x_{i} .
\end{aligned}
$$

Let $S$ be the sum of $0,1, \cdots, L_{T}-1$ so that

$$
S=\frac{L_{T}\left(L_{T}-1\right)}{2} \text {. }
$$

When $L_{T}$ is even, $L_{T}-1$ is odd and

$$
S \not \equiv 0 \bmod \left(L_{T}\right) \text {. }
$$

Also

$$
S=\sum_{i=0}^{L_{T}-1} x_{i}=\sum_{i=0}^{L_{T}-1} y_{i}
$$

Therefore

$$
\sum_{i=0}^{L_{T}-1} z_{i}=\sum_{i=0}^{L_{T}-1}\left(y_{i}-x_{i}\right)+\sum_{i: y_{i}<x_{i}} L_{T} \equiv 0 \bmod \left(L_{T}\right) .
$$

If $z_{i}$ 's are distinct then

$$
\sum_{i=0}^{L_{T}-1} z_{i}=S
$$


From (17) and (18) it follows that

$$
S \equiv 0 \bmod \left(L_{T}\right) .
$$

This contradicts (15) and hence $z_{i}$ s are not all distinct. So a third code cannot be formed when the length of the code is even.

Example 1: T/S AML codes for $L_{T}=5, W=5$ are as follows:

$\left[\begin{array}{l}10000 \\ 10000 \\ 10000 \\ 10000 \\ 10000\end{array}\right]\left[\begin{array}{l}10000 \\ 01000 \\ 00100 \\ 00010 \\ 00001\end{array}\right]\left[\begin{array}{l}10000 \\ 00100 \\ 00001 \\ 01000 \\ 00010\end{array}\right]\left[\begin{array}{l}10000 \\ 00010 \\ 01000 \\ 00001 \\ 00100\end{array}\right]\left[\begin{array}{l}10000 \\ 00001 \\ 00010 \\ 00100 \\ 01000\end{array}\right]$.

\section{PERFormance ANAlysis OF T/S AML CODES}

In an asynchronous, incoherent FO-CDMA using ON-OFF keying only bit " 1 " is encoded by the CDMA sequence. To analyze the performance of FO-CDMA a simple protocol is considered in which the transmission between any transmitter and receiver is pairwise and continuous and that the receivers are already synchronized with the transmitters. An ideal link is considered in which the performance deterioration is only due to the multiple access interference (MAI) and the receiver noises, i.e., shot noise and thermal noise are ignored. All receivers are assigned codes which have equal weight and length. All transmitters use identical incoherent sources of the same wavelength, power and are modulated at the same bit rate using the same signal format. The signal at the receiver contains the desired user's signal $b$ and the interference signal, $I . I$ is the sum of all cross correlations which overlap in time at that receiver.

1) Probability Density Function for Interference Signal at a Receiver: When any two T/S codes of the same family having equal number of pulses/row, $p$, are correlated there are $p^{2}$ possible overlaps in any one row. Therefore, total number of overlaps over all the rows $R$ is $R p^{2}$. Then the probability of overlap for T/S code is $R p^{2} / L_{T}$ and for T/S AML code it is $R / L_{T}$. It is assumed that binary " 1 " or " 0 " is sent with equal probability. In ON-OFF keying only bit " 1 " is encoded by a CDMA sequence. For T/S AML code the probability of overlap in case of oN-OFF keying is $R / 2 L_{T}$ and the complement of this event, i.e., the probability of no overlap for T/S AML is $\left(1-R / 2 L_{T}\right)$. In writing this, we have assumed that either there is complete overlap of chips or there is no overlap. For $\mathrm{T} / \mathrm{S}$ AML codes the probability density function $P_{I}$ for interference signal $I$ for the chip synchronous case is given by

$$
P_{I}=\sum_{i=0}^{N-1}\left(\begin{array}{c}
N-1 \\
i
\end{array}\right)\left(\frac{R}{2 L_{T}}\right)^{i}\left(1-\frac{R}{2 L_{T}}\right)^{N-1-i} \delta(I-i) \text {. }
$$

In a FO-CDMA system to evaluate the exact $P_{I}$ at a receiver the knowledge of $N-1$ probability density functions are needed and to evaluate the performance at all receivers $N(N-1) / 2$ probability density functions are required [8]. When $N$ is large the task of evaluating $N(N-1) / 2$ probability density functions is tedious. Hence the upper bound on the probability of error is evaluated by considering the chip synchronous case.
2) Probability of Error/Bit When Chips Are Synchronous: The exact probability of error/bit, $P_{e}$, at a receiver is defined as

$$
\begin{aligned}
P_{e}(\text { exact })= & P_{r}(I \geq T h / b=0) \cdot P_{r}(b=0) \\
& +P_{r}(I<T h / b=1) \cdot P_{r}(b=1) .
\end{aligned}
$$

Because a FO-CDMA system with incoherent signal processing is a positive system, no errors can occur when the desired user transmits a " 1 ," as the signal at the receiver (with or without interference signal $I$ ) will be at least equal to the threshold value.

In (20), the bit error is only due to the first term [8] and is given by

$$
P_{e}(\text { exact })=\frac{1}{2} \int_{T h}^{\infty} P_{I} d_{I}
$$

$P_{e}$ for T/S AML code is given by

$$
P_{e}=\frac{1}{2} \sum_{i=T h}^{N-1}\left(\begin{array}{c}
N-1 \\
i
\end{array}\right)\left(\frac{R}{2 L_{T}}\right)^{i}\left(1-\frac{R}{2 L_{T}}\right)^{N-1-i} .
$$

$P_{e}$ (when chips are synchronous) for OOC is given by [1]

$$
P_{e}=\frac{1}{2} \sum_{i=T h}^{N-1}\left(\begin{array}{c}
N-1 \\
i
\end{array}\right)\left(\frac{W^{2}}{2 L_{T}}\right)^{i}\left(1-\frac{W^{2}}{2 L_{T}}\right)^{N-1-i} .
$$

The number of OOC's that can be obtained for a given $L_{T}$ and $W$ is given by

$$
N \leq \frac{L_{T}-1}{W(W-1)}
$$

In (22), $R=W$ as there is only one pulse/row in a $\mathrm{T} / \mathrm{S}$ AML code. It can be seen from (22) and (23) that for a given $P_{e}, N$ and $W, L_{T}$ in the case of T/S AML code is $R$ times smaller than that of OOC.

Let $L_{T_{1}}$ be the minimum required temporal length for given $N$ and $W$ as calculated by (24) for OOC's. It is assumed that it is possible to design the codes with the minimum temporal length given by (24). Let $L_{T_{2}}$ be the minimum temporal length required for the same $N$ and $W$ to achieve $10^{-9}$ error performance as calculated by (23). It is found that $L_{T_{2}}>L_{T_{1}}$ for $W \leq 8$ and $N>20$ and also $L_{T_{1}}>L_{T_{2}}$ for $W \geq 9$ and $N>20$. Since both conditions have to be met, i.e., it should be possible to design the OOC's and also $10^{-9}$ error performance is required, the larger value of $L_{T}$ is considered. In the case of OOC's it is found by calculations that when the above two conditions are satisfied $L_{T}$ is minimum when $W=9$ for $N>20$.

From (22) the values of $L_{T}$ required to achieve $10^{-9}$ error performance are calculated for T/S AML codes and for OOC's from (23) and (24). For $W=810$ ( $W=R$ in the case of $\mathrm{T} / \mathrm{S}$ AML code) and $P_{e}=10^{-9}$ the temporal lengths required for T/S AML codes and OOC's are shown in Fig. 2. From Fig. 2, it can be seen that trade off between the temporal lengths and spatial lengths can be made for given $N$ and $P_{e}$ for T/S AML codes.

In Fig. 3, the capacities of 1-D FO-CDMA network employing OOC's and 2-D T/S SPR network employing T/S AML 


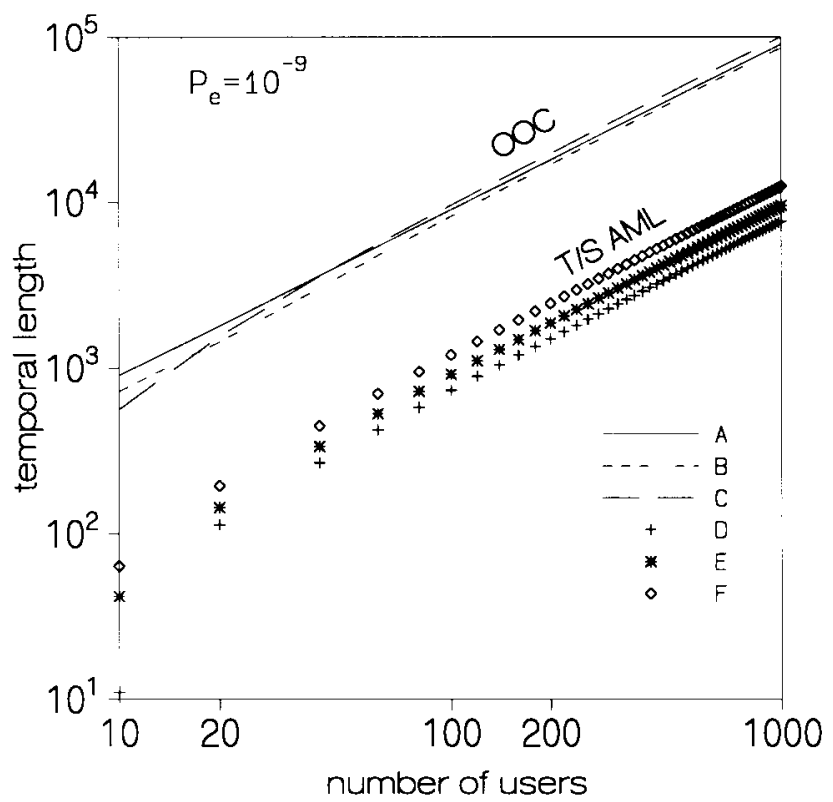

Fig. 2. Temporal lengths for OOC and T/S AML codes. A: $W=10$, OOC; $\mathrm{B}: W=9$, OOC; C: $W=8$, OOC; $\mathrm{D}: W=10$, T/S AML; E: $W=9$, T/S AML; F: $W=8$, T/S AML.

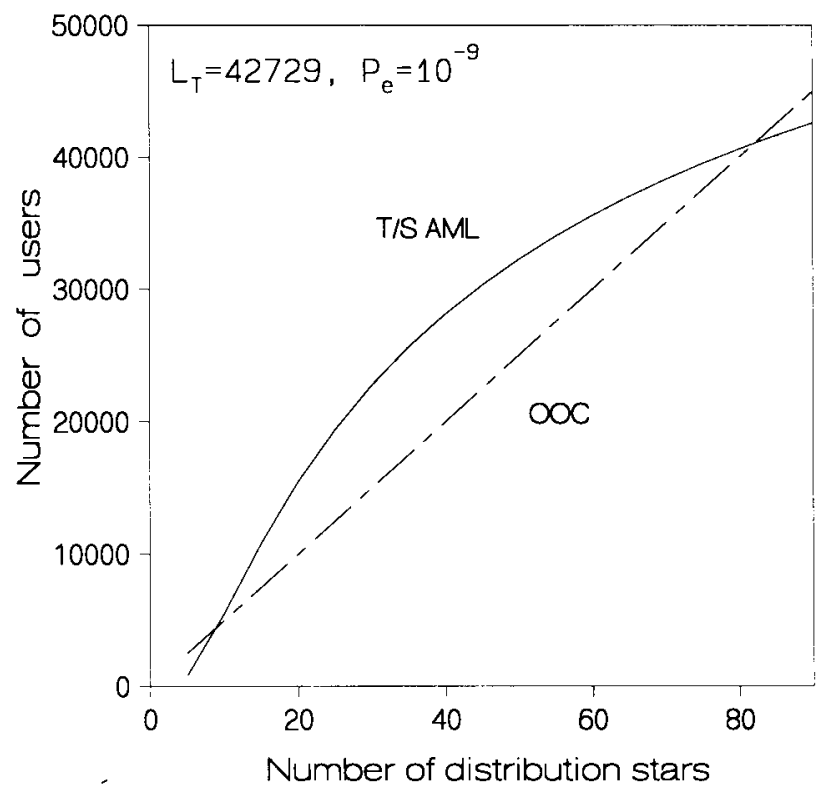

Fig. 3. Capacities of 2-D networks employing T/S AML codes and a 1-D network employing OOC's.

codes are shown for temporal length equal to 42729 and error performance $10^{-9}$. In the 1-D network the number of users supported is equal to 500 on one distribution star for the above values of $L_{T}$ and $P_{e}$. In Fig. 3, in the 1-D network the users on different distribution stars will not be able to communicate with each other. Let us compare the values of $N$ in 1- and 2-D networks as the number of distribution stars are varied. In a 1-D network, the number of users supported on $x$-distribution stars is equal to $500 x$. For the same values of $L_{T}$ and $P_{e}, N$ for the 2-D network is higher than that of the 1-D network when the number of distribution stars is above 10 and below

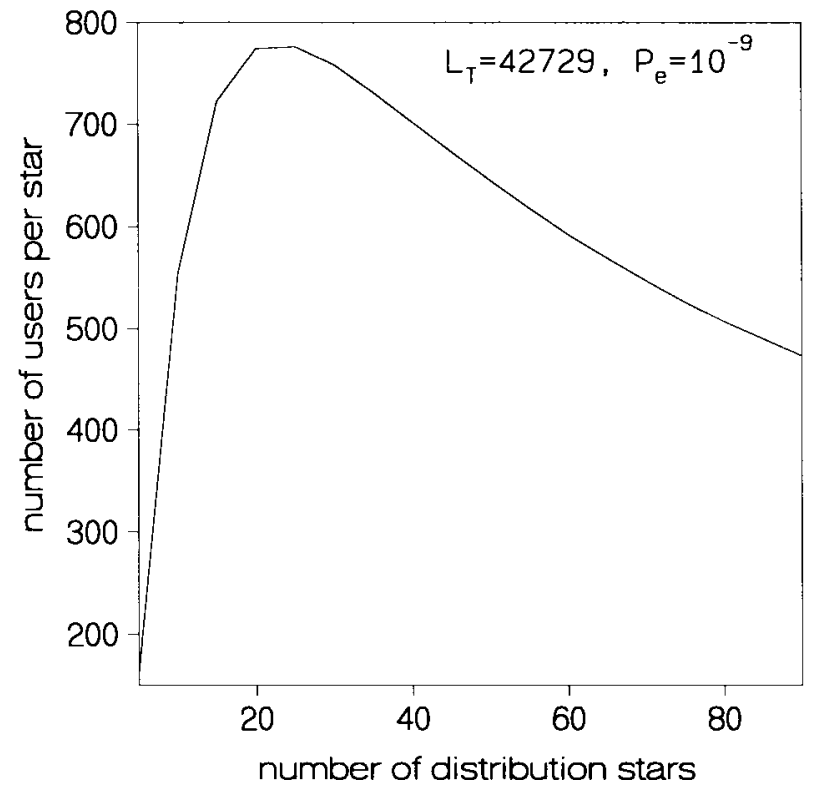

Fig. 4. Number of users supported/star in a 2-D network employing T/S AML codes. For the same temporal length and $P_{e}$, a 1-D network employing OOC's supports 500 users.

80. In the 2-D network, all users will be able to communicate with each other unlike in the 1-D case.

Alternatively, the number of users supported/distribution star in a 2-D network employing T/S AML codes is shown in Fig. 4 for the same values of $L_{T}$ and $P_{e}$ as in Fig. 3. N/star in the case of OOC is equal to 500 whereas for T/S AML codes it is a maximum of 776 when the number of spatial channels is equal to 25 .

It can be shown that SPR T/S codes perform better than $\mathrm{T} / \mathrm{S}$ codes with multiple-pulses-per-row for the same weight. Probability of interference for T/S codes with $p$ pulses per row is $R p^{2} / L_{T}$. Probability of interference is minimum when $p=1$. Threshold is $R p=$ constant if $W$ is constant. Hence, as the number of pulses/row increases the probability of interference also increases.

3) Analysis Using Optical Hardlimiter: For a given $P_{e}$, $N, W$, and $L_{T}$ can be further reduced by reducing the MAI [8]. One way to reduce the interference effect is by placing an optical hardlimiter before the TDL. An ideal optical hardlimiter is defined as

$$
\begin{aligned}
q(x) & =1, & & x \geq 1 \\
& =0, & & 0 \leq x<1 .
\end{aligned}
$$

The hardlimiter acts as an intensity dependent bistable device. When the optical intensity $x$ is greater than or equal to one, the hardlimiter will limit the intensity to one and if the intensity is less than " 1 " the response of the hardlimiter will be " 0. ." For example, an interference pattern of [2003001000] will have a maximum of " 6 " at the output of a correlator whereas after clipping the pattern will be [1001001000] and the peak value is " 3 ."

$P_{e}$ for a 2-D network employing T/S AML codes when hardlimiter is introduced in the decoder is derived as follows: Assume that the desired user is sending " 0 " and $i$ other users 


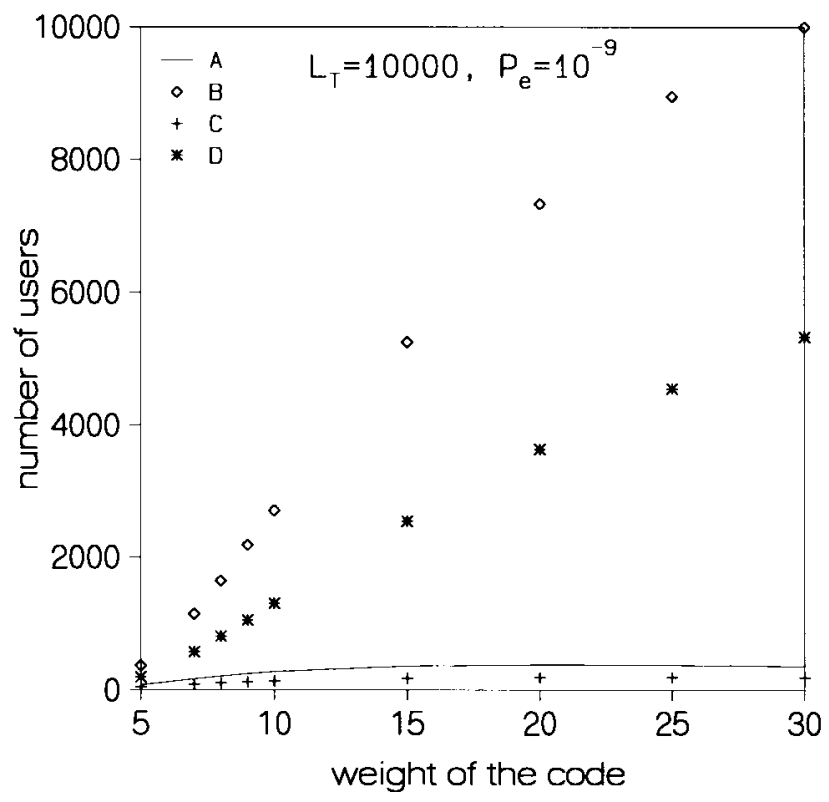

Fig. 5. Capacities of 1-D networks employing OOC's and 2-D networks employing T/S AML network with and without hardlimiter. A: 1-D, hardlimiter; B: 2-D, hardlimiter; C: 1-D without hardlimiter; D: 2-D without hardlimiter.

are sending " 1 " and the threshold is set equal to the weight of the code. An error occurs if atleast $W$ users are contributing $W$ interferences at different taps on the same tapped delay line. Therefore, there are $\left(\begin{array}{c}i \\ W\end{array}\right)$ ways of choosing the interference causing users, and $W$ ! ways of choosing the interferences on different taps of the same TDL. The probability that an user contributes an interference to a particular tap is $1 / L_{T}$. Therefore, the probability of error is upper bounded by $P_{e} \leq$ $\frac{1}{2} \sum_{i=W}^{N-1} \operatorname{Pr}$ (false detection/ $i$ other users are sending 1$) \times \operatorname{Pr}(i$ other users are sending 1)

$$
P_{e} \leq \sum_{i=W}^{N-1}\left(\frac{1}{2}\right)^{N}\left(\begin{array}{c}
N-1 \\
i
\end{array}\right)\left(\begin{array}{c}
i \\
W
\end{array}\right) W !\left(\frac{1}{L_{T}}\right)^{W} .
$$

$P_{e}$ for OOC is given by [9]

$$
P_{e} \leq \sum_{i=W}^{N-1}\left(\frac{1}{2}\right)^{N}\left(\begin{array}{c}
N-1 \\
i
\end{array}\right)\left(\begin{array}{c}
i \\
W
\end{array}\right) W !\left(\frac{W}{L_{T}}\right)^{W} .
$$

Fig. 5 shows the capacities of a 2-D network employing T/S AML codes with hardlimiter and without hardlimiter for $L_{T}=10000$ and $P_{e}=10^{-9}$. In the same plot are also shown the capacities of a 1-D network employing OOC's with hardlimiter and without hardlimiter for the same values of $L_{T}$ and $P_{e}$.

\section{EXPERIMENTAL RESULTS}

1) $T / S$ AML codes: A temporal/spatial single-pulse-perrow prototype network with two optical encoders and one optical decoder was built to test the autocorrelation and crosscorrelation properties of T/S AML codes. Schematic diagram of the prototype network built is as shown in Fig. 6.

Encoders/decoders were built using $4 \times 4$ couplers and fiber-optic delay coils. Four $2 \times 2$ couplers were used as distribution stars. Graded index 50/125 $\mu$ m mulitmode fiber

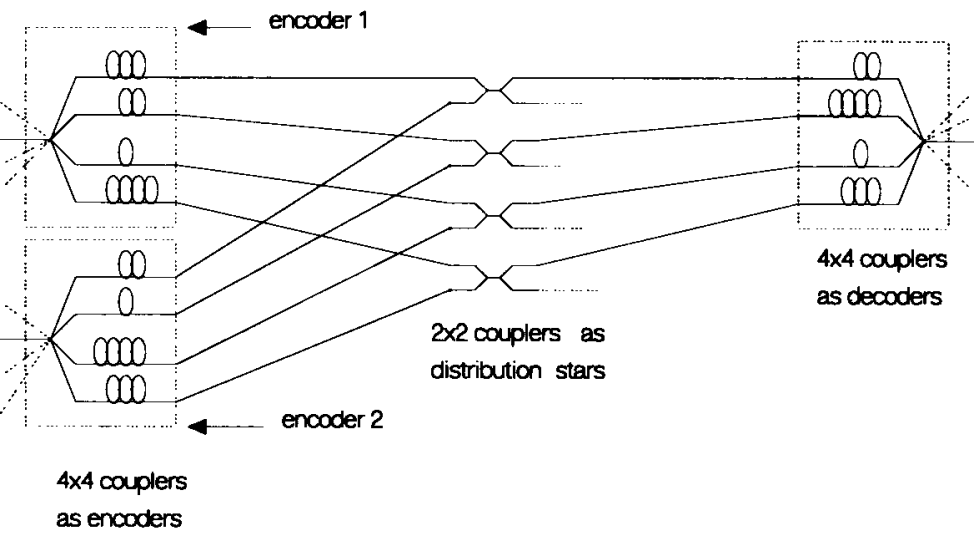

Fig. 6. A T/S SPR prototype network with two encoders and one decoder.
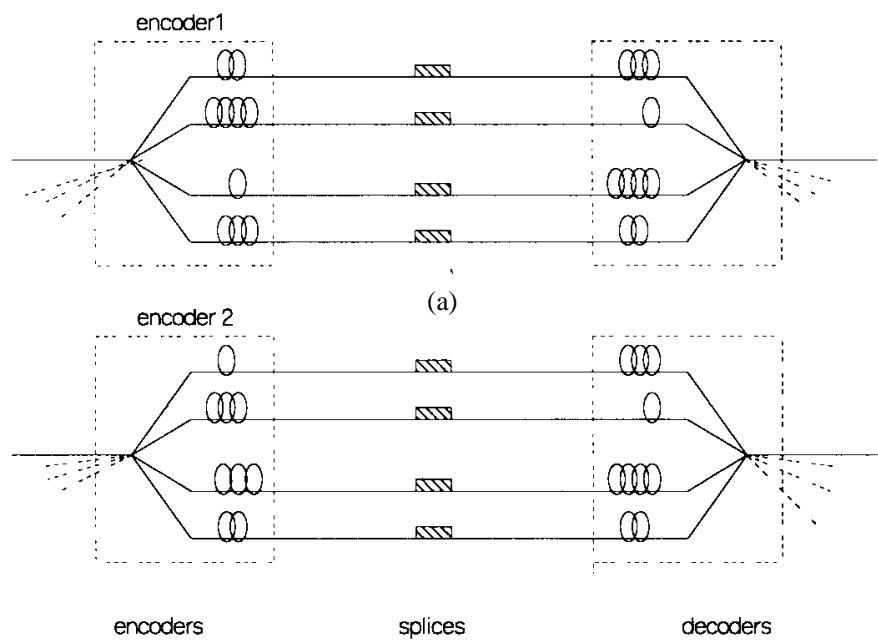

(b)

Fig. 7. (a) Encoder and matched decoder for autocorrelation testing. (b) Encoder and unmatched decoder for cross-correlation testing.

was used for the fabrication of multimode couplers and the delay coils. Encoders 1 and 2 were built to generate codes 1 and 2, respectively. Codes 1 and 2 are as follows:

$$
\begin{aligned}
& \text { code } 1 \quad \text { code } 2 \\
& {\left[\begin{array}{l}
10000 \\
01000 \\
00100 \\
00010
\end{array}\right]\left[\begin{array}{l}
10000 \\
00100 \\
00001 \\
01000
\end{array}\right] .}
\end{aligned}
$$

Since the weight of the codes is $4,1 \times 4$ couplers were required to build encoders. We have used $4 \times 4$ couplers in which three inputs in each coupler are dummy as shown in Fig. 6. To connect to encoders and one decoder the size of the distribution star required is $2 \times 1$. We have used $2 \times 2$ couplers, where one end in each coupler is dummy as shown in dotted lines in Fig. 6. The decoder is matched to code 1 and hence gives autocorrelation for encoder 1 output. Cross correlation is obtained for encoder 2 output as it is not matched to code 2 .

A $1300-\mathrm{nm}$ laser diode with a peak power of $0.5 \mathrm{~mW}$ was used to generate optical pulses. An Anritsu O/E converter with Ge APD and of sensitivity $-30 \mathrm{dBm}$ was used for detecting the output signal. Multimode couplers were fabricated in the laboratory by fusion method using pen torch flame. Each $4 \times 4$ 


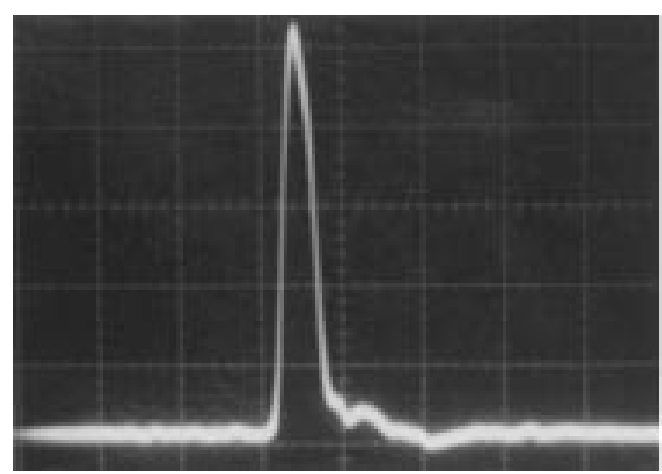

(a)

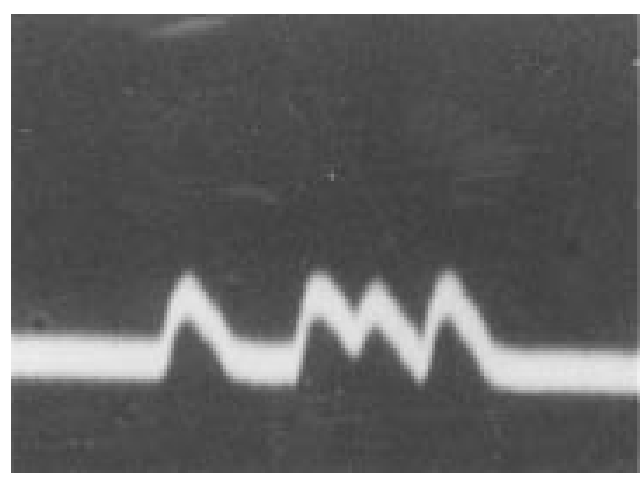

(c)

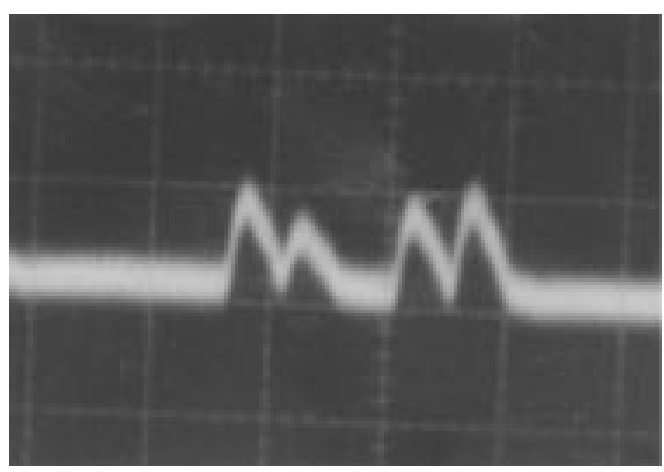

(e)

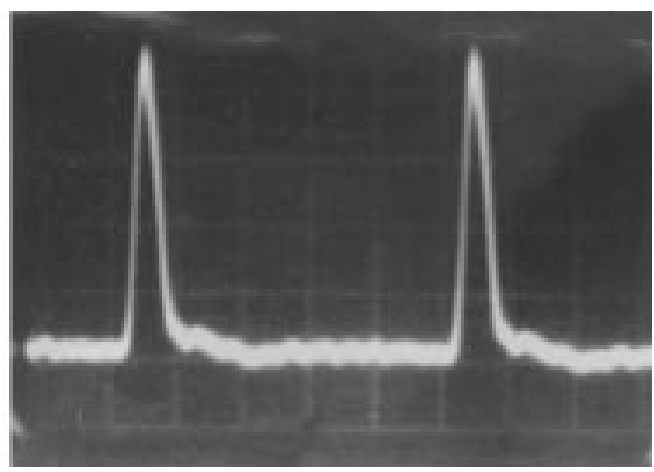

(b)

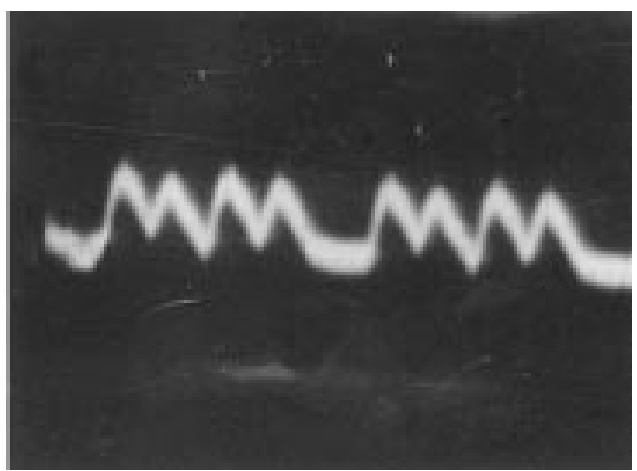

(d)

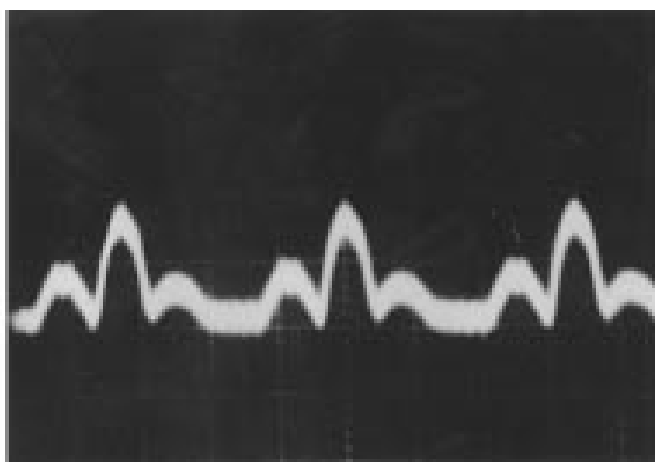

(f)

Fig. 8. (a) Autocorrelation of T/S AML code for "010" bits. (b) Autocorrelation of T/S AML code when a continuous steam of ones are input. (c) Cross correlation of T/S AML codes for "010" bits. (d) Cross correlation of T/S AML codes when a continuous stream of ones are input. (e) Cross correlation of Park et al. codes for "010" bits. (f) Cross correlation for Park et al. codes when a continuous stream of " 1 "'s are input.

and $2 \times 2$ coupler had an excess loss of 5-6 dB. Since the endto-end loss between encoder input to the decoder output was high, the experimental setup was modified as shown in Fig. 7. Four $2 \times 2$ distribution stars were removed and the encoder 1 was directly spliced to decoder as shown in Fig. 7(a). Another decoder (similar to the one in Fig. 6) was built. Encoder 2 was spliced to this decoder as shown in Fig. 7(b).

Pulses of $10 \mathrm{~ns}$ width at a repetition rate of $50 \mathrm{~ns}$ from a pulse generator were used for driving the laser diode. A single pulse was input to encoder 1 and the autocorrelation output at the decoder is as shown in Fig. 8(a). Encoder 1 was driven with a series of $10 \mathrm{~ns}$ width pulses at a repetition rate of $50 \mathrm{~ns}$. Fig. 8(b) shows the autocorrelation for two such frames. To encoder 2, an optical pulse was input and the cross-correlation output is as shown in Fig. 8(c). A repetitive pattern of "11110" was obtained when a continuous stream of pulses were input to encoder 2. Fig. 8(d) shows the cross correlation of two such repetitive patterns. From Fig. 8(c) and (d), it may be seen that the cross-correlation peak has a " 1 " which agrees with the result proved in Section III.

2) Codes of Park et al. [6]: Park et al. codes were tested for their cross-correlation property. Autocorrelation of these codes is similar to that of Fig. 8(a) and (b) [6]. The delay coils in the encoder and decoder of Fig. 7(b) were changed so as to generate code 1 and decode code 2, respectively. Codes 1 and 2 are as follows:

$$
\begin{aligned}
& \text { code } 1 \quad \text { code } 2 \\
& {\left[\begin{array}{l}
1000 \\
0100 \\
0001 \\
0010
\end{array}\right]\left[\begin{array}{l}
0100 \\
0001 \\
0010 \\
1000
\end{array}\right] .}
\end{aligned}
$$


Fig. 8(e) shows the cross correlation when a single pulse was input to the encoder. In Fig. 8(e), the difference in the heights of the pulses is due to unequal division of optical power in the input coupler. When a continuous stream of pulses was input to the encoder a repetitive cross-correlation pattern of " 0121 " was obtained. Fig. 8(f) shows the cross-correlation output of the decoder for three such frames. It may be seen that a peak cross correlation is obtained for Park et al. codes.

\section{CONCLUSIONS}

One-dimensional codes with low cross correlation and autocorrelation designed for FO-CDMA networks have the disadvantage that the length of the code increases rapidly as the number of users or the weight is increased. To overcome this problem, 2-D codes were designed and demonstrated. But the cross-correlation values of these 2-D codes is high.

In this paper, we have constructed 2-D codes with minimal out-of-phase autocorrelation and cross-correlation properties. The construction of these codes is much simpler unlike the design of optical orthogonal codes which have the minimal out-of-phase autocorrelation and cross-correlation properties for 1-D codes. We have constructed temporal/spatial singlepulse-per-row codes using the operation of addition modulo an integer and these codes are named temporal/spatial addition modulo $L_{T}$ (T/S AML) codes.

Temporal lengths required for T/S AML codes is much smaller than that of 1-D codes (OOC's) having the same crosscorrelation properties for a given error performance. Hence, a 2-D single-pulse-per-row network employing T/S AML codes will have better error performance. It is shown that the capacity of 2-D networks employing T/S AML codes is higher than that of a 1-D networks employing OOC's. By using an optical hardlimiter in the decoder the improvement in the capacities of 1-D and 2-D networks is shown.

A T/S SPR prototype network with optical encoding and decoding using tapped delay lines is built to test the autocorrelation and cross-orrelation properties of T/S AML codes and Park et al. codes. The experimental results show that T/S AML codes have out-of-phase autocorrelation " 0 " and crosscorrelation "1." Experimental results for Park et al. codes show that cross correlation is two.

From the above results it can be concluded that 2-D networks employing T/S AML codes have nearly ideal correlation properties and are well suited for fiber-optic LAN's.

\section{REFERENCES}

[1] J. A. Salehi, "Code division multiple access techniques inoptical fiber networks Part I: Fundamental principles," IEEE Trans. Commun., vol. 37, pp. 824-833, Aug. 1989.

[2] A. A. Shaar and P. A. Davies, "Prime sequences: Quasi optimal sequences for channel code division multiplexing," Electron. Lett., vol. 19, pp. 888-889, Oct. 1983.

[3] A. S. Holmes and R. R. Syms, "All optical CDMA using quasi prime codes,” J. Lightwave Technol., vol. 10, pp. 279-286, Feb. 1992.

[4] S. V. Maric, Z. I. Kostic, and E. L. Titelbaum, "A new family of optical orthogonal sequences for use in spread spectrum fiber optic local area networks," IEEE Trans. Commun., vol. 41, pp. 1217-1221, Aug. 1993.
[5] S. V. Maric, "New family of algebrically designed optical orthognal codes for fiber optic CDMA networks," Electron. Lett., vol. 29, pp. 538-539, Mar. 1993.

[6] E. Park, A. J. Mendez, and E. M. Gasmeiere, "Temporal/spatial loptical CDMA networks: Design, demonstration and comparison with temporal network," IEEE Photon. Technol. Lett., vol. 4, pp. 1160-1162, Oct. 1992.

[7] A. J. Mendez, J. L. Lambert, J. M. Morookian, and K. M. Gagliardi, "Synthesis and demonstration of higher speed bandwidth efficient optical code division multiple access (CDMA) tested at $1 \mathrm{~Gb} / \mathrm{sec}$ throughput," IEEE Photon. Technol. Lett., vol. 6, pp. 1146-1149, Sept. 1994.

[8] J. A. Salehi and C. A. Brackett, "Code division multiple access techniques in optical fiber networks Part II: Systems and performance analysis," IEEE Trans. Commun., vol. 37, pp. 834-842, Aug. 1989.

[9] F. R. K. Chung, J. A. Salehi, and V. K. Wei, "Optical orthogonal code design analysis and application," IEEE Trans. Inform. Theory, vol. IT-35, pp. 595-604, May 1989.

[10] E. S. Shivaleela and A. Selvarajan, "Temporal/spatial fiber optic CDMA network," in Proc. First Nat. Conf. Commun., NCC'95, Kanpur, India, Mar. 13-14, 1995, pp. 28-31.

[11] E. S. Shivaleela, Kumar N. Sivarajan, and A. Selvarajan, "Tempo$\mathrm{ral} / \mathrm{spatial}$ codes for asynchrous fiber optic CDMA network: Design and performance," in Proc. Second Nat. Conf. Commun., NCC'96, Bombay, India, Feb. 16-18, 1996, pp. 23-26.

E. S. Shivaleela received the B.E. degree in electronics and communication from the Government BDT College of Engineering, Davanagere, Mysore University, in 1987. She received the M.Sc.(Eng.) degree in electrical communication engineering from the Indian Institute of Science, India, in 1997.

She is presently working as a Scientific Officer in the Department of Electrical Communication Engineering, Indian Institute of Science, Bangalore, India. Her interests are mainly in the areas of all-optical networks and fiber and integrated optic components for photonic gigabit systems.

Kumar N. Sivarajan (S'88-M'91) received the B.Tech. degree in electrical engineering (electronics) from the Indian Institute of Technology, Madras, India, in 1987 and the M.S. and Ph.D. degrees in electrical engineering from the California Institute of Technology, Pasadena, in 1988 and 1990, respectively.

From 1990 to 1994, he was with the IBM Thomas J. Watson Research Center, Yorktown Heights, NY. Since October 1994, he has been with the Indian Institute of Science, Bangalore, India. He was an Academic Visitor with the IBM Thomas J. Watson Research Center during the summers of 1996 and 1997. His research interests are in the design, architecture, and performance analysis of optical, cellular, and ATM networks. He has coauthored a book (with R. Ramaswami) on optical networks.

Dr. Sivarajan is an Editor of the IEEE/ACM TRANSACTIONS ON NETWORKING and an Associate of the Indian Academy of Sciences. He was the recipient of the IEEE Charles LeGeyt Fortescue Fellowship for the year 1987-1988, corecipient (with R. Ramaswami) of the IEEE Communications Society 1996 William R. Bennett Prize Paper Award, and corecipient (with R. Ramaswami) of the 1997 IEEE W. R. G. Baker Prize.

A. Selvarajan received the M.Sc. degree in physics from Annamalai University and the Ph.D. degree from the Indian Institute of Science, Bangalore, India.

Currently, he is a Professor and Chairman at the Department of Electrical Communication Engineering, Indian Institute of Science. He has more than 150 publications in the areas of optics and spectroscopy, holography, acoustooptics, fiber optics, integrated optics and optical communications. He has been a Visiting Scientist at Optical Sciences Center, University of Arizona, Tempe, University of Upsala, Sweden, Technical University of Denmark, University College London, U.K., and International Centre for Theoretical Physics, Italy.

Dr. Selvarajan is a member of the SPIE, the International Society for Optical Engineering, Optical Society of India, and the Institution of Electronics and Telecommunication Engineers (IETE). 